\title{
Power-Control and Rate Selection Medium Access Control for Full-duplex Asymmetric Transmission
}

\author{
Yan Chen, Yanjing Sun*, Qi Cao, Haiwei Zuo \\ the School of Information and Control Engineering, China University of Mining and Technology \\ Xuzhou, Jiangsu, China 221116 \\ chyan@cumt.edu.cn; yjsun@cumt.edu.cn; qcao@cumt.edu.cn; zuohaiwei123@126.com
}

\begin{abstract}
Full-duplex asymmetric transmission can improve the system throughput and transmission fairness effectively. However, in the real WIFI network, the length of downlink packet is usually much longer than that of uplink, which can decrease the system performance. In this paper, a WIFI network with a full-duplex access point (FD_AP) and half-duplex stations is studied and a full-duplex Medium Access Control (MAC) protocol based on power control and rate selection (PCRS) is proposed to reduce the effect of Inter Client Interference (ICI) and improve the system throughput and transmission fairness. Furthermore, the differences in packet length and minimum sensitivity are taken into consideration. Simulation results show that PCRS MAC protocol can achieve higher throughput, as compared to the current Distributed Coordination Function (DCF) and a simple fullduplex MAC protocol without PCRS. PCRS MAC also maintains a high level of fairness.
\end{abstract}

\section{KEYWORDS}

full-duplex, asymmetric, packet length, power-control and rate selection, MAC.

\section{ACM Reference format:}

Yan Chen, Yanjing Sun*, Qi Cao, Haiwei Zuo, 2017. Power-Control and Rate Selection Medium Access control for Full-duplex asymmetric transmission. In Proceedings of 10th EAI International Conference on Mobile Multimedia Communications, Chongqing, China, July 13-14 (MOBIMEDIA 2017), 5 pages.

DOI: $10.1145 /$ nnnnnnn.nnnnnnn

\section{INTRODUCTION}

In current half-duplex Wireless Local Area Network (WLAN), the adopted MAC protocol is Distributed Coordination Function (DCF), which is contention-based. When the number of stations is

Permission to make digital or hard copies of all or part of this work for personal or classroom use is granted without fee provided that copies are not made or distributed for profit or commercial advantage and that copies bear this notice and the full citation on the first page. To copy otherwise, to republish, to post on servers or to redistribute to lists, requires prior specific permission and/or a fee.

MOBIMEDIA 2017, July 13-14, Chongqing, People's Republic of China Copyright ( 2017 EAI 978-1-63190-156-0
RTS/CTS frame. Besides, DCF gives all nodes in the network the same chance of transmission. However, in the real WIFI network all stations serve for uplink and only AP serves for downlink. Therefore, in a saturated network DCF may result in much smaller opportunity of downlink transmission than uplink transmission. Furthermore, the downlink traffic from AP is much heavier than uplink traffic from each station [1]. Therefore, when the number of stations is increased, DCF can hardly meet the demand of real network.

Full-duplex wireless communication has been proved to be feasible in recent years [2], and relevant researches have been carrying out. While employing full-duplex radio in WIFI, two basic transmission modes exist: symmetrical transmission (Fig. 1 (a)) and asymmetric transmission (Fig. 1 (b)). Both stations and $\mathrm{AP}$ are required to have full-duplex capability when using bidirectional transmission while only AP required when asymmetric transmission is employed. So we can only upgrade AP in the existing WIFI networks to increase the chance of downlink transmission which hence can greatly improve the system performance.

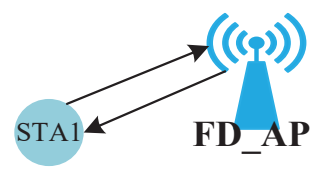

(a)

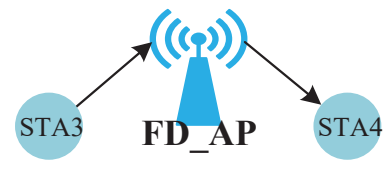

(b)
Fig. 1: The basic transmission modes of full-duplex (a) symmetrical transmission (b) asymmetric transmission.

Apart from self-interference (SI), the Inter-Client Interference (ICI) in full-duplex asymmetric transmission is also a significant impediment. The blue circle in Fig. 2 represents the transmission range of full-duplex AP (FD_AP). Assume STA3 has a packet to transmit and wins the channel and FD_AP has packet transmit to STA4, signals from STA3 will cause interference at STA4. If STA3 and STA4 are close to each other, the ICI is very strong and STA4 cannot receive information from FD_AP correctly.

In the past two years, there are two main approaches dominating in relevant research. In [3], the AP and uplink transmitter control their transmit power to let FD_AP and downlink receiver have the same maximum Signal to Interference 
plus Noise Ratio (SINR), so that the sum rate of uplink and downlink can be maximized. In [4], packet-alignment-based capture effect was explored to help station ignore collision because they can decode one packet correctly.

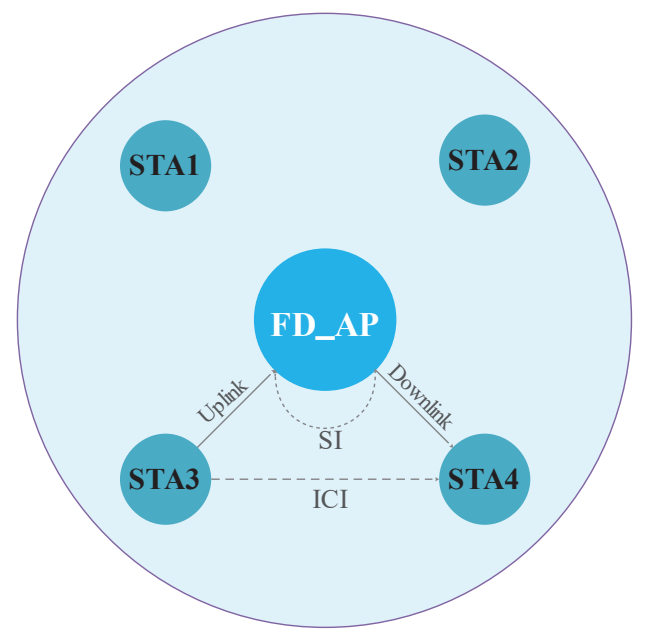

Fig. 2: Full-duplex Asymmetric transmission with SelfInterference (SI) and Inter-Client Interference (ICI).

Several full-duplex MAC protocols [3]-[7] have been proposed to support asymmetric transmission. However, they consider the same length on uplink packets and downlink packets, which is almost impossible because the length of the downlink packet is usually much longer than that of uplink in practice [1]. Besides, in most of these researches, link rates were set to constant. However, In order to ensure that the system has a $10^{-5}$ order of bit error rate after a certain rate is selected, the received signal strength must be higher than a certain value, which is called minimum Sensitivity and had been proposed in IEEE 802.11 standard. Table 1 shows the minimum sensitivity corresponding to the modulation mode at different rates of $20 \mathrm{MHZ}$ bandwidth specified by 802.11 . In the transmission process, due to noise and channel fading and other factors, the receiver side is only able to acquire a small signal, so the transmission links must be matched with an appropriate transmission rate.

Table 1. Minimum Sensitivity required in $\mathbf{8 0 2 . 1 1}$

\begin{tabular}{cccc}
\hline $\begin{array}{c}\text { Rate } \\
\text { (Mbps) }\end{array}$ & $\begin{array}{c}\text { Modulation/ } \\
\text { Coding }\end{array}$ & $\begin{array}{c}\text { Minimum } \\
\text { Sensitivity } \\
\text { (dBm) }\end{array}$ & $\begin{array}{c}\text { SINR(dB) } \\
\text { N=-90dBm }\end{array}$ \\
\hline 6 & BPSK 1/2 & -82 & 5 \\
\hline 9 & BPSK 3/4 & -81 & 8 \\
\hline 12 & QPSK 1/2 & -79 & 10 \\
\hline 18 & QPSK 3/4 & -77 & 13 \\
\hline 24 & 16QAM 1/2 & -74 & 16 \\
\hline 36 & 16QAM 3/4 & -70 & 19 \\
\hline 48 & 64QAM 1/2 & -66 & 22 \\
\hline 54 & 64QAM 2/3 & -65 & 25 \\
\hline
\end{tabular}

Therefore, these schemes can hardly get the same performance as expected when employed in the real WIFI network. In this paper, we propose a full-duplex MAC protocol based on power control and rate selection (PCRS) in the presence of ICI to improve throughout and transmission fairness. Power control and rate selection are employed to reduce ICI and transmission time. We take the difference between the packet length of uplink and downlink into consideration as well as the effect of minimum sensitivity.

The rest of the paper is organized as follows. Our proposed idea is described in Section 2. Section 3 is the PCRS MAC protocol. Section 4 shows simulation results for proposed fullduplex MAC protocol. Conclusions are shown in Section 5.

\section{SYSTEM MODEL}

In this paper, we consider a full-duplex WIFI network where the AP supports full-duplex and all stations are solely half-duplex enabled.

In Fig. 2, we assume STA3 wins the channel, AP can use its full-duplex capability for downlink data transmission to the target node STA4. In this case, the signal from STA3 interferes STA4. When positions of STA4 and STA3 are fixed, ICI is reduced if STA3 transmits with a lower power. The total duration of asymmetric transmission lies on the downlink data rate because the length of the downlink packet is much longer than that of uplink, so we can reduce the rate of uplink without changing the total transmit time of the system. Also when a certain rate is selected, we only need to ensure that the received signal strength at receiver is higher than Minimum Sensitivity. Therefore, we can reduce the rate and power of STA3 to reduce the ICI and consequently to improve the SINR, thus we can select a higher rate for downlink transmission.

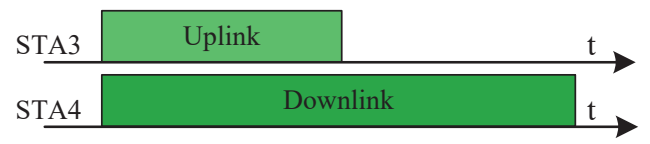

(a)

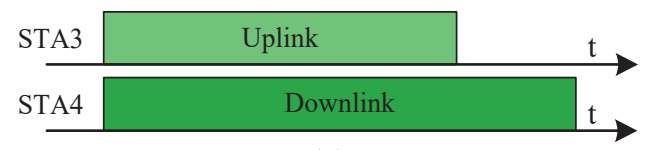

(b)

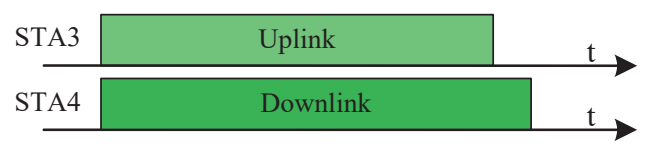

(c)

Fig. 3: An illustration for the change of total transmission time with the change of rate and power (a) transmission with original rate (b) reduce rate of STA3 (b) reduce rate and power of STA3 and increase rate of STA4.

We use Fig. 3 to illustrate our motivation. STA3 $\rightarrow$ AP is uplink and $\mathrm{AP} \rightarrow \mathrm{STA} 4$ is downlink, besides, the packet length of 
Power-Control and Rate Selection Medium Access Control for Full-duplex Asymmetric Transmission

AP is much longer than STA3. Fig. 3(a) shows that STA3 transmits information with the highest rate it can achieve. With the effect of ICI, downlink transmission either transmit at a low rate or fail to build reliable link, so we conceive downlink transmit time is much longer than uplink transmit time. Then, as Fig. 3(b) shows that we only reduce the rate of STA3, uplink transmit time will increase but is still shorter than downlink transmit time. At Last, the transmit power of STA3 can be reduced accordingly. Then the ICI will be suppressed and AP has a chance to select a higher rate for downlink transmission, which shortens total transmission time. This can be seen from Fig. 3(c).

In this paper, we try to maximize the system throughput by rate selection and power control. In a transmission process, the size of the transmitted packet is fixed, so when the total transmission time is the shortest, the throughput reaches the maximum.

$L_{u}, L_{d}$ are the packet length of uplink and downlink. $P_{i \rightarrow j}$ denotes the received signal strength that client $j$ received from client $i . R$ is a set of available rates. $r$ is the selected rate. $\left(r_{d}, r_{u}\right)$ is the selected optimum rate pair based on the rate selection mechanism. In this paper, a $T x$ is the station for uplink, and $R x$ is the station receiving downlink signals. $N$ denotes background noise. The system throughput $\mathrm{S}$ can express as

$$
\mathrm{S}=\frac{L_{d}+L_{u}}{T_{\text {total }}}=\frac{L_{d}+L_{u}}{T_{\text {control }}+\max \left(L_{d} / r_{d}, L_{u} / r_{u}\right)},
$$

Where $T_{\text {control }}$ is the time used to transmit control frames. Therefore, the purpose of this paper is to find the rate pair that minimizes the total asymmetric transmission time:

$$
\begin{aligned}
& \left(r_{d}, r_{u}\right)=\underset{r_{d} \in R, r_{u} \in R}{\arg \min }\left(\max \left(L_{d} / r_{d}, L_{u} / r_{u}\right)\right) \\
& P_{T x} \leq P_{\max } \\
& \text { s.t. } \quad r_{d} \geq 0, r_{u} \geq 0 \\
& L_{d} / r_{d} \approx L_{u} / r_{u}
\end{aligned}
$$

The rate pair of uplink and downlink depends on the SINR of received signal.

$$
\begin{aligned}
& r_{d}=P D R\left(R, S I N R_{d}\right) \\
& r_{u}=P D R\left(R, S I N R_{u}\right)
\end{aligned}
$$

SINR of $R x$ and AP are given by,

$$
\begin{gathered}
S I N R_{d}=\frac{P_{A P \rightarrow R x}}{P_{T x \rightarrow R x}+N} \\
\operatorname{SINR}_{u}=\frac{P_{T x \rightarrow A P}}{S I+N} .
\end{gathered}
$$

Here, the Packet Delivery Rate $\operatorname{PDR}(R, \operatorname{SINR})$ [8], as a function of rate set $R$ and the SINR, finds the highest rate can be reached. Table. 1 shows the relationship between rate and SINR we employed. In order to ensure downlink reaching the highest rate, the transmit power of $\mathrm{AP}$ is set to be equal to the maximum power. The power of $T x$ is the only transmit power to be controlled. The indoor path loss in channel model is given by
MOBIMEDIA 2017, July 13-14, Chongqing, China

$$
P L(d)=P L\left(d_{0}\right)+10 \times n \times \log \left(d / d_{0}\right)[d B],
$$

where we set $d_{0}=1 \mathrm{~m}, n=3$ and let $P L(1)=48 d B$.

\section{PCRS MAC PROTOCOL}

PCRS MAC protocol is based on RTS/CTS mechanism. In current RTS/CTS MAC protocol, some information including packet length, received signal strength, the modulation mode and link rate can be known to receiver after RTS and CTS are successfully transmitted.

To build PCRS MAC protocol, some information is added into control frame. Three way handshaking [6] is a useful way for information exchange. In PCRS, we let all stations adopt DCF to contend for uplink channel.

Fig. 4 demonstrates the PCRS MAC protocol and the detailed procedure of $T x, R x$, and AP under the protocol are described as follows.

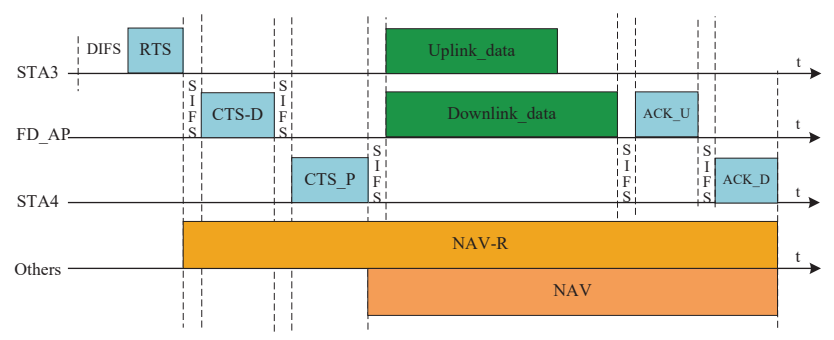

(a)

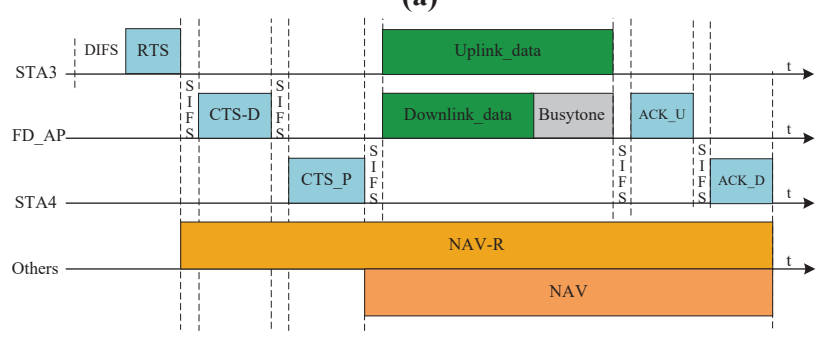

(b)

Fig. 4: PCRS MAC protocol (a) downlink transmission time longer than uplink transmission time (b) uplink transmission time longer than downlink transmission time

- $\quad$ STA3 has a packet to transmit and channel is sensed idle for more than a DIFS. Meanwhile, AP has a packet to transmit to STA4.

- $\quad$ STA3 sends a RTS frame with maximum transmit power to compete for channel.

- $\quad$ After receiving the RTS frame from STA3, AP calculates the link rate that uplink can achieve and STA4 is selected as the $R x$, Then AP transmits CTS-D frame including the address of STA4.

- $\quad$ After receiving RTS and CTS-D frame, STA4 calculates the optimal rate pair for uplink and downlink. Then STA4 packages rate pair to CTS-P frame and transmit it after SIFS time delay. 
- Once CTS-P frame is received, AP starts downlink transmission with $r_{u}$ and STA3 starts uplink transmission with $r_{d}$ and corresponding transmit power after SIFS delay.

- When AP finishes transmitting and receiving data, it transmits ACK-U frame to STA3.

- After receiving data successful and overhearing ACK-U frame from AP, STA4 transmits ACK-D frame to AP.

- $\quad$ After overhearing ACK-U and ACK-D frames, all stations and AP return to initial state. Otherwise, AP or STA3 return to initial state for retransmission.

- Stations that have packets to transmit and back off timers return to zero will contend for channel after channel is sensed idle for more than a DIFS.

Note that all control frames must transmit with the basic rate and the maximum power to ensure receiver can fully obtain channel state information and packet information. When downlink transmit time is shorter than uplink transmit time, AP should insert Busytone signal (Fig. 4(b)) after data packet to prevent contention by hidden stations. Besides, if $R x$ is too close to $T x$, asymmetric transmission is impossible. In this situation, we let $T x$ transmit data at highest rate that can be reached.

To finish PCRS, the control frame structure must be changed. First, CTS-D requires extra 8 bytes of CTS frame, where 6 bytes represent downlink address and 2 bytes represent packet length. Second, 6 bits are inserted into CTS-P to represent link rate pair.

\section{PERFORMANCE EVALUATION}

In order to evaluate our MAC protocol, we simulate our MAC using MATLAB. A wireless network with a full-duplex AP and half-duplex stations are build up. The stations are uniformly and randomly distributed in a circle area around AP. Fig. 5 is a typical realization with 20 stations.

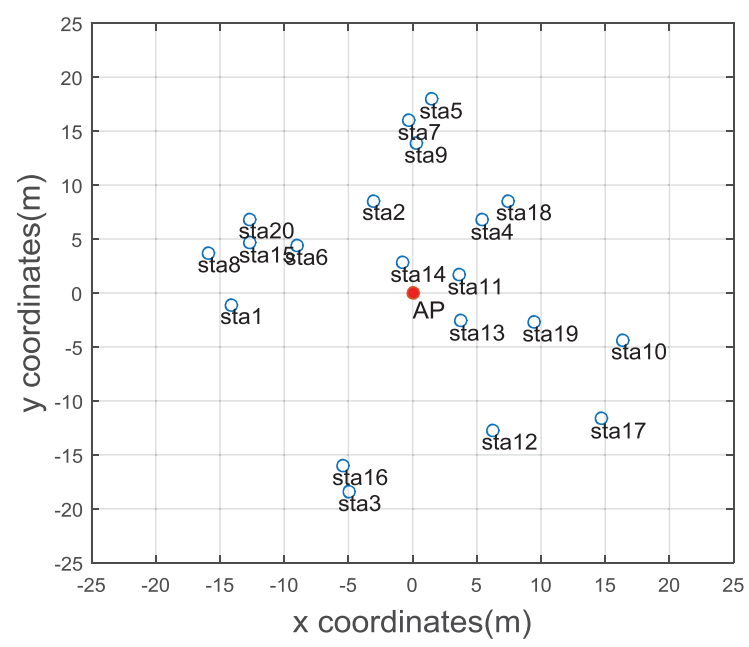

Fig. 5: A typical random topology with 20 stations.

We consider a saturated network to evaluate PCRS MAC. Also, 802.11 DCF and full-duplex MAC without PCRS are simulated with the same parameters. The system parameters are selected according to Table 2. Besides, half-duplex DCF protocol with same packet length are also simulated to show the effect caused by the difference of the packet length between uplink transmission and downlink transmission, where we set the packet size to be 1500 bytes.

Table 2: Comparison of Coefficients from Atomistic.

\begin{tabular}{cc||cc}
\hline Parameter & \multicolumn{1}{c|}{ Value } & Parameter & Value \\
\hline PHY_header & 128bit & Ra & $20 \mathrm{~m}$ \\
\hline RTS & 160 bit+PH & W0 & 32 \\
\hline CTS-D & 112 bit+PH+64bit & $\mathrm{m}$ & 6 \\
\hline CTS-P & 112 bit+6bit+PH & Pmax & $20 \mathrm{dBm}$ \\
\hline ACK & 112 bit+PH & SIC & $110 \mathrm{~dB}$ \\
\hline SIFS & $16 \mathrm{us}$ & DIFS & $34 \mathrm{us}$ \\
\hline Uplink & $100 / 300 / 500$ bytes & Downlink & $\begin{array}{c}1500 / 11454 \\
\text { bytes }\end{array}$ \\
\hline
\end{tabular}

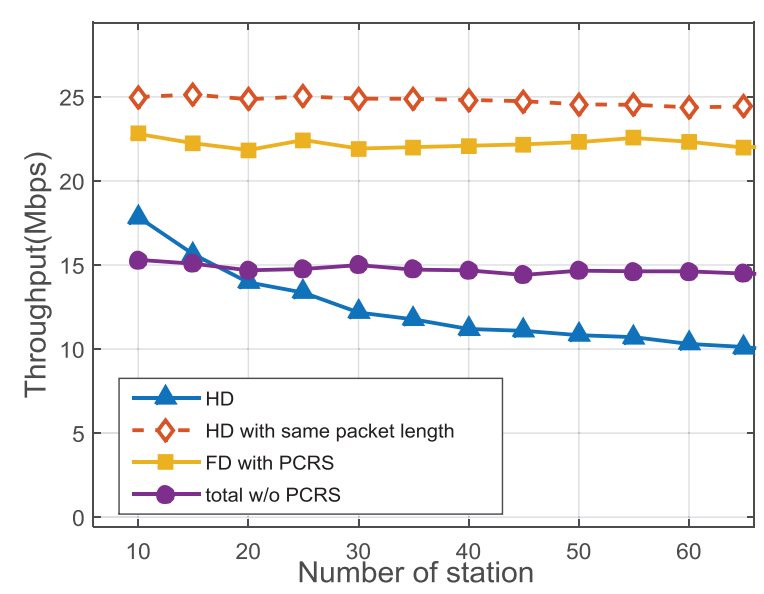

Fig. 6: Average throughput with respect to the number of stations.

Fig. 6 shows the system throughput with respect to the number of stations. From the figure, we have following findings.

(1) Half-duplex transmission with the equal packet length can achieve $98.8 \%$ and $150 \%$ higher throughput than that with non-equal length data frame for 10 stations and 60 stations. Besides, the throughput of half-duplex transmission with equal packet length decreases slightly with the number of stations increased, which support the analysis in the literature [8]. The throughput of half-duplex transmission with non-equal packet length decreases $40 \%$ when the number of stations increases from 10 to 60 . This is due to the fact that the overhead of the control frames used for transmitting a large number of shorter uplink data frames is increased and the number of collisions increases as the number of stations rises. 
Power-Control and Rate Selection Medium Access Control for Full-duplex Asymmetric Transmission

(2) The simple full-duplex MAC without PCRS can achieve higher throughput than DCF when there are more than 20 stations. This is because that small number of nodes contribute to less collision the system conflict and the use of full-duplex will increase the overhead of MAC control frame. Without power control, full-duplex transmission becomes less.

(3) PCRS MAC protocol improves throughput by $30.5 \%$ and $125 \%$ over DCF for 10 stations and 60 stations, and by $53 \%$ over simple full-duplex MAC without PCRS. Besides, the throughput of PCRS is roughly invariant to the number of stations.

We also use the fairness index that was proposed in [10] to evaluate the fairness in throughput among stations. Fig. 7 indicates the fairness index versus the number of stations. As the number of stations increases, the average ICI increase and downlink transmission rate of half-duplex decreases. Therefore, fairness index for all the schemes are degraded and fairness index for half-duplex downlink throughput decrease even faster, which cause the fairness index for system throughput of half-duplex decreases faster than PCRS. As result shows that fairness index for both uplink, downlink and system throughput of PCRS are higher than 0.97, which implies that all stations almost have the same transmission opportunity.

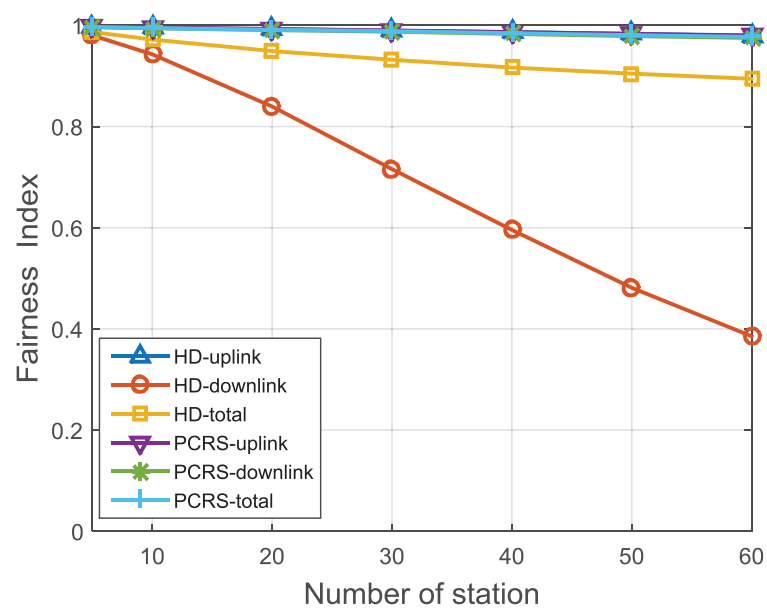

Fig.7: Fairness index with respect to the number of stations.

\section{CONCLUSIONS}

In this paper, we proposed a MAC protocol for asymmetric fullduplex transmission using power control and rate selection to reduce ICI and improve downlink transmission opportunity. Simulation result shows that PCRS MAC can achieve higher and more stable system throughput and can give more fair transmission opportunity for all nodes than that of half-duplex.

\section{ACKNOWLEDGMENTS}

This work is supported by National Natural Science Foundation of China (51504255, 51504214), the Fundamental Research and
MOBIMEDIA 2017, July 13-14, Chongqing, China

Development Foundation of Jiangsu Province (BE2015040), the Transformation Program of Scientific and Technological Achievements of Jiangsu Provence (Sub project) under Grant (BA2012068), Natural Science Foundation of Jiangsu province of China (BK20130199, BK20131124), the Fundamental Research Funds for China University of Mining and Technology (2014ZDPY16).

\section{REFERENCES}

[1] Palit R, Naik K, Singh A. 2012. Anatomy of WiFi access traffic of smartphones and implications for energy saving techniques. International Journal of Energy, Information and Communications 3, 1 (2012), 1-16

[2] Bharadia D, Mcmilin E, Katti S. 2013. Full duplex radios. Computer Communication Review 43, 4 (2013), 375-386. DOI: https://doi.org/10.1145/2 486001.2486033

[3] Choi W, Lim H, Sabharwal A. 2015. Power-Controlled Medium Access Control Protocol for Full-Duplex WiFi Networks IEEE Transactions on Wireless Communications 14, 7 (2015), 3601-3613. DOI: https://doi.org/10.1109/TWC.2 015.2408338

[4] Tang A, Wang X. 2015. A-Duplex: Medium Access Control for Efficient Coexistence Between Full-Duplex and Half-Duplex Communications. IEEE Transactions on Wireless Communications 14, 10 (2015), 5871-5885. DOI: https://doi.org/10.1109/TWC.2015.2443792

[5] Shih-Ying Chen, Ting-Feng Huang, Kate Ching-Ju Lin, Y.-W. Peter Hong, Ashutosh Sabharwal. 2017. Probabilistic Medium Access Control for Full Duplex Networks With Half-Duplex Clients. IEEE Transactions on Wireless Communications. 16, 4 (2017), 2627-2640.

[6] Haiwei Zuo, Yanjing Sun, Changlin Lin, Song Li, Hongli Xu, Zefu Tan, and Yanfen Wang. 2016. A Three-way Handshaking Access Mechanism for Point to Multipoint In-band Full-duplex Wireless Networks. KSII Transactions on Internet and Information Systems 10, 7 (2016), 3131-3149. DOI: http://dx.doi.o $\mathrm{rg} / 10.3837 /$ tiis.2016.07.015

[7] Chulmin Kim, Cheeha Kim. 2016. A full duplex MAC protocol for efficient asymmetric transmission in WLAN. In Computing, Networking and Communications (ICNC'16). IEEE, Hawaii, HI,USA, 1-5.

[8] Daniel Halperin, Wenjun Hu, Anmol Sheth, and David Wetherall, 2010. Predictable 802.11 packet delivery from wireless channel measurements. In Proceedings of the ACM SIGCOMM 2010 conference (SIGCOMM'10). ACM, New York, NY, USA, 159-170.

[9] Bianchi G. 2000. Performance analysis of the IEEE 802.11 distributed coordination function. IEEE Journal on selected areas in communications 18, 3 (2000), 535-547. DOI: https://dx.doi.org/10.1109/49.840210

[10] Jain R, Chiu D M, Hawe W R. A quantitative measure of fairness and discrimination for resource allocation in shared computer system. Hudson, MA: Eastern Research Laboratory, Digital Equipment Corporation, 1984. 